\title{
Las políticas alimentarias de México: un análisis de su marco regulatorio*
}

\author{
Food Policy in Mexico: An Analysis of the Legal Framework
}

As políticas alimentares do México: uma análise de seu marco regulatório

\author{
RICARDO LÓPEZ SALAZAR ${ }^{* *}$ \\ ENRIQUe DaVID GaLlaRdo García ${ }^{* \star \star}$
}

FeCha de ReCePCión: 26 de AGosto de 2014. FeCha de ACEPTACIÓN: 12 De nOVIembre de 2014

Doi: dx.doi.org/10.12804/esj 17.01.2014.01

Para citar este artículo: López Salazar, R. y Gallardo García, E. D. (2015). Las políticas alimentarias de México: un análisis de su marco regulatorio. Estudios Socio-Jurídicos, 17(1), 11-39. Doi: dx.doi.org/10.12804/esj 17.01.2014.01

\section{RESUMEN}

Este artículo tiene como objetivo analizar el marco regulatorio de las políticas alimentarias de México y, en particular, se orienta a discutir sobre la distribución de competencias entre los gobiernos federal, estatal y municipal. Los hallazgos indican que existe una clara indefinición normativa de las políticas alimentarias de México, ya que no se cuenta con herramientas suficientes para asegurar que todos los mexicanos puedan acceder a una alimentación mínima. En la parte de la distribución de competencias, el Gobierno Federal ha monopolizado las acciones relacionadas con la producción de alimentos, mediante lo estipulado en el Plan Nacional de Desarrollo, aunque sin una implementación efectiva, por lo que es altamente recomendable la elaboración de un plan nacional orientado a potenciar la producción y distribución de alimentos en México, que establezca de manera clara las directrices entre los tres órdenes de gobierno.

Palabras clave: Políticas alimentarias, marco jurídico, competencias, órdenes de gobierno, México.

* Artículo de reflexión financiado por Conacyt para el desarrollo de la investigación doctoral de los autores, en el Centro de Investigación en Alimentación y Desarrollo y en el Instituto Tecnológico y de Estudios Superiores de Monterrey, México.

** Doctor en Ciencias con especialidad en Desarrollo Regional por el Centro de Investigación en Alimentación y Desarrollo (CIAD). Profesor investigador titular C de la Universidad Autónoma de Ciudad Juárez, Chihuahua, México. Correo electrónico: ricardo.lopez@uacj.mx

*** Doctor en Políticas Públicas por la Escuela de Graduados en Administración Pública y Política Pública del Tecnológico de Monterrey (EGAP). Profesor investigador de la Universidad del Istmo, Campus Ixtepec, Oaxaca, México. Correo electrónico: edavidgallardog@outlook.com 


\section{ABSTRACT}

This article aims to analyze the legal framework for food policy in Mexico in particular aims to discuss the distribution of powers and capabilities between the federal, state and municipal government. The findings indicates that there is a clear legal limbo of food policies of Mexico and not have enough tools to ensure that all Mexicans have access to a minimum food levels. In the part of the distribution of capabilities, the federal government has monopolized the actions related to food production through the provisions of the National Development Plan but without effective implementation. So it is highly recommended the development of a project to enhance the production and distribution of food in Mexico, which clearly establishes guidelines to follow among the three levels of government that favors the acquisition of better results in the food issue.

Key words: Food policy, legal framework, capabilities, levels of government, México.

\section{RESUMO}

Este artigo tem como objetivo analisar o marco regulatório das políticas alimentares do México e em particular orienta-se a discutir sobre a distribuição de competências entre o governo federal, estatal e municipal. Os resultados indicam que existe uma clara indefinição normativa das políticas alimentares do México devido a que não se tem ferramentas suficientes para assegurar que todos os mexicanos possam aceder a uma alimentação mínima. Na parte da distribuição de competências, o governo federal tem monopolizado as ações relacionadas com a produção de alimentos através do estipulado no Plano Nacional de Desenvolvimento ainda sem uma implementação efetiva do mesmo. Por isto, é altamente recomendável a elaboração de um plano nacional orientado a potenciar a produção e distribuição de alimentos no México, que estabeleça de forma clara as diretrizes a seguir entre as três ordens de governo.

Palavras-chave: políticas alimentares, marco jurídico, competências, ordens de governo, México. 


\section{Introducción}

El crecimiento de la pobreza alimentaria en los últimos años en México ha otorgado un nuevo impulso al debate sobre la efectividad de los programas sociales dedicados a su combate y erradicación. La discusión se ha centrado en analizar programas asistenciales alimentarios como Oportunidades o el Programa de Abasto Alimentario (PAL), y sectoriales dedicados a fortalecer la producción agrícola, como Procampo. Sin embargo, poco se ha profundizado en el estudio de las leyes que sustentan la aplicación de las políticas alimentarias del país y, sobre todo, se desconoce si existe un marco regulatorio que involucre los diferentes poderes y órdenes de gobierno, en el cual se establezcan claramente las bases y los mecanismos de colaboración, así como de la participación de los ciudadanos y los actores públicos.

El objetivo del artículo es analizar las leyes y los planes que regulan la aplicación de la política alimentaria mexicana, como la Constitución Política de los Estados Unidos Mexicanos, la Ley General de Desarrollo Social, la Ley General de Desarrollo Sustentable, el Plan Nacional de Desarrollo (2007-2012), algunas reglas de operación, así como otras leyes secundarias que abordan aspectos de la política alimentaria del país. En particular, se analizan las competencias y los mecanismos de colaboración entre los órdenes de gobierno en la materia.

El trabajo se estructura en cuatro partes. En la primera se analizan las distintas políticas alimentarias implementadas por el gobierno federal de México. La segunda aborda constitucionalmente la política alimentaria y su vinculación con el Plan Nacional de Desarrollo 2006-2012. En la tercera parte se identifican los elementos que regulan la política alimentaria en las leyes General de Desarrollo Social y de Desarrollo Rural Sustentable. Finalmente, se hace un apartado de conclusiones sobre los temas expuestos.

\section{Programas alimentarios y políticas asistenciales para la seguridad alimentaria en México a partir de la década de los ochenta}

Al inicio de la década de los ochenta, el Estado mexicano inició diversas medidas orientadas a la disminución drástica de su papel en la economía 
que impulsaron la desgravación de tarifas arancelarias para la apertura del mercado interno; la privatización de la mayoría de las empresas públicas; la liberalización de los flujos de inversión, tanto de inversión extranjera directa como de cartera o especulativa, y la liberalización del sistema financiero y su reprivatización. No obstante, los resultados indican que en poco más de tres décadas de cambios y reformas estructurales el crecimiento de la economía ha resultado ser insuficiente, inestable y excluyente. El crecimiento económico promedio desde 1981 fue de apenas el 0,72\% (tabla 1), muy lejano del $7 \%$ estimado para disminuir el desempleo, la pobreza, la informalidad y algunos otros rezagos sociales y económicos.

Tabla 1. Tasa de crecimiento del producto interno bruto en México (1961-1980 y 1981-2010)

\begin{tabular}{|c|c|c|}
\hline Periodo & $1961-1980$ & $1981-2010$ \\
\hline TCPA (\%) & 3,67 & 0,72 \\
\hline
\end{tabular}

Fuente: Elaboración propia con información del Banco Mundial (2010).

De acuerdo con Zapata, lo que se puede constatar a partir de la década de los ochenta es una desarticulación entre "la estrategia de acumulación y el marco de regulación institucional" $(2005$, p. 25), a partir de lo cual ya no es posible pensar las acciones económicas en función de los intereses de los actores sociales de la producción, como son los empresarios y los trabajadores; hecho que marca una notable diferencia con lo que fue el papel del Estado-nación durante el modelo de sustitución de importaciones. Los intereses políticos del Estado se han separado cada vez más de la idea de conformar un modelo de desarrollo con una sociedad nacional integrada, autónoma y políticamente fortalecida. Todo ello parece indicar que la transnacionalización del mercado interno se ha convertido más en una política de Estado, aun cuando con ello se reducen fuertemente los márgenes de maniobra de las autoridades políticas y se tiende a incrementar la vulnerabilidad macroeconómica.

En este escenario de cambios estructurales y relativa estabilidad macroeconómica, los programas de asistencia social y las políticas alimentarias se han visto poco favorecidos. La excepción la marca quizá el final del sexenio de José López Portillo (1976-1982), cuando se aprecia uno de los intentos 
más serios por garantizar una alimentación digna a la población mexicana. Durante esa administración se instauró el Sistema Alimentario Mexicano ${ }^{1}$ (SAM) con el propósito de promover la autosuficiencia alimentaria, y con ello atacar los problemas del hambre, la pobreza, la salud y la desnutrición de los estratos marginados de la sociedad mexicana. Con este propósito se impulsaron dispositivos de asistencia técnica, financiamiento oportuno, capacitación y transferencia de tecnología, inversión en infraestructura hidráulica, de almacenamiento, procesamiento, empaque y transporte; así como la instrumentación de un sistema de distribución y abasto de alimentos. Se establecieron programas de apoyo a la agricultura de temporal, se impulsó la agroindustria campesina y se otorgaron subsidios generalizados y dirigidos para facilitar el acceso a la tierra, a la tecnología y a los insumos. Asimismo, se desarrollaron mecanismos de detección de zonas críticas con alta prevalencia de desnutrición, y se establecieron programas de orientación alimentaria a la población (SAM, 1980).

Parte de su relativo éxito radica quizás en haber incorporado una visión particular del problema alimentario, muy diferente a las concepciones todavía prevalecientes en la década de los setenta, que atribuían a los factores técnicos, climáticos y de recursos naturales las causas principales del origen de este. Con el SAM se generó la convicción de que la producción y transformación de bienes agrícolas de origen animal y vegetal, así como la industria de bienes de capital e intermedios y la distribución de alimentos, ya no podía ser abordada por separado. Estos sectores empezaron a ser considerados parte de una unidad estructural amplia en la cual los conceptos de cadena y sistema alimentario se constituyeron en una nueva forma de abordar el problema de la alimentación y en una condición para alcanzar la autosuficiencia alimentaria.

Para algunos analistas (Meseguer, 1998), el SAM representó el único intento de planeación estratégica más o menos exitoso en materia de política social alimentaria concebido en las últimas décadas, ya que logró asegurar una articulación favorable entre la producción, acopio, transformación, abasto y consumo de alimentos mediante una coordinación eficaz de los

El Gobierno Federal creó el SAM en marzo de 1980, año durante el cual se estima que cerca de 24 millones de personas (35\% del total) gastaban más del 60\% de sus ingresos en la compra de alimentos. 
programas de política social y económica. Por desgracia, la abrupta caída de los precios internacionales del petróleo en 1982 redujo el ingreso de divisas en perjuicio del presupuesto federal y ello ocasionó el retiro de recursos a dicho programa.

Posteriormente, durante el gobierno de Miguel de la Madrid, las políticas alimentarias revistieron una naturaleza distinta. Se abandonó el enfoque de la autosuficiencia, y el abastecimiento de alimentos pasó a depender, en gran parte, del comercio internacional. El Programa Nacional de Alimentación, operado por la Comisión Nacional de Alimentación, fue el instrumento por medio del cual se dirigieron las nuevas acciones estratégicas de política económica y social. Los programas relacionados con producción, abasto, consumo, salud y nutrición quedaron a cargo de organismos que operaban sin la menor coordinación real, de tal manera que la política social solo era reconocible a través de la retórica del discurso del gobierno y por la operación de programas clientelares de reparto de alimentos (Meseguer, 1998). Fue el caso del Desarrollo Integral de la Familia, que operaba el Programa de Asistencia Social Alimentaria, supuestamente para apoyar a la población de escasos recursos económicos, en particular a aquellas familias con deficiencias alimentarias y nutricionales.

El Plan Nacional de Desarrollo (PND) de ese sexenio establecía, en materia de salud y nutrición, el impulso de la planta productiva de alimentos básicos, así como el apoyo y los subsidios para el abaratamiento de productos altos en proteínas. ${ }^{2}$ No obstante, estos últimos fueron objetados por funcionarios de gobierno, quienes veían en esa práctica un "ejercicio insano de populismo" que impedía la acción de la economía de libre mercado. La regulación de la demanda y la transferencia de ingresos - que hasta entonces había sido una característica de la intervención estatal- quedaron sujetas a los criterios de control presupuestario de la Secretaría de Hacienda. A partir de entonces, la política alimentaria nacional se enfocó en la compra de alimentos requeridos sin importar su origen, quién y cómo se produjeran, lo que significó cambiar el concepto de autosuficiencia alimentaria por el de soberanía alimentaria (Vázquez, Ortiz y Montes, 2005).

El nuevo enfoque, sin embargo, no resultó compatible con el discurso de las reformas estructurales impulsadas durante el gobierno de Salinas de

2 Véase en el sitio de internet http://www.edomexico.gob.mx (mayo de 2006). 
Gortari. En su lugar se propuso una estrategia de "seguridad alimentaria", cuyo fin consistía en garantizar la disponibilidad de alimentos mediante su importación, sin reparar en su volumen. Para hacer operativa dicha estrategia se continuó con el Programa Nacional de Alimentación, pero ahora dividido en dos apartados: el Sistema Nacional de Seguridad Alimentaria y el Sistema Nacional de Vigilancia Alimentaria y Nutricional. Estos, junto con el Programa Nacional de Solidaridad (Pronasol), constituyeron la clave de la política social de atención a los pobres, que incluía todas las acciones que las instituciones del Estado habían desarrollado décadas atrás, como el establecimiento de tiendas rurales, lecherías, despensas, subsidio a la tortilla y desayunos escolares. Como parte de los lineamientos generales de estos programas, al final del sexenio operaban en el país 23 programas menores de asistencia alimentaria que ejercían en conjunto un presupuesto cercano a los 2.000 millones de dólares.

La atención que durante ese sexenio se le dio al problema alimentario se explica por la influencia que tuvieron en los años ochenta las recomendaciones de política económica del Banco Mundial y el Fondo Monetario Internacional, así como el concepto de seguridad alimentaria promovido por la Organización de las Naciones Unidas para la Agricultura y la Alimentación (FAO, por su sigla en inglés). Ya desde 1983 se había planteado como finalidad estratégica de los diferentes gobiernos garantizar a toda la población el acceso material y económico a los alimentos básicos. Para ello se debían incorporar políticas económicas orientadas al desarrollo agrícola y rural, así como distintos mecanismos de estabilidad interna y macroeconómica, y una mayor participación en el comercio mundial, a fin de lograr la suficiencia, la estabilidad y la seguridad de los suministros alimentarios.

No obstante, al final del sexenio, bajo los lineamientos de dicha estrategia, la propuesta económica del gobierno redujo significativamente la capacidad de reproducción interna de la agricultura mexicana, sobre todo la autosubsistencia de la economía campesina de las zonas marginadas, misma que se vio fuertemente afectada por el retiro generalizado de subsidios, la liberación del precio de los alimentos y la reducción de los apoyos a la canasta básica. Adicionalmente, el fracaso de los mecanismos de política social y la inoperancia de los sistemas de abasto de alimentos a las zonas marginadas se vio reflejado, entre otros aspectos, en una creciente concentración del ingreso. 
Los saldos desfavorables del gobierno salinista no impidieron, sin embargo, continuar con la estrategia de seguridad alimentaria. En el sexenio de Ernesto Zedillo, esta se concretó, inicialmente, en el Programa de Alimentación y Nutrición Familiar, dado a conocer el 16 de febrero de 1995, a partir de tres ejes fundamentales: a) desayunos escolares, b) canasta básica alimentaria para las familias más pobres del medio rural y c) canasta y apoyos a familias de áreas urbanas marginadas. Con la idea de duplicar las metas cuantitativas del sexenio anterior, algunas de las acciones particulares del programa consistieron en repartir un millón de despensas familiares al mes y dos millones de desayunos escolares en las zonas de mayor marginación. Asimismo, se proponía combatir la desnutrición en las comunidades indígenas, rurales y zonas urbanas marginadas facilitando el acceso a una canasta básica alimentaria a partir de una erogación mayor de recursos en subsidios y programas de asistencia social.

A inicios de 1996, el Programa de Alimentación y Nutrición Familiar fue sustituido por el Programa de Alimentación Salud y Educación, que en esencia mantenía los mismos objetivos; pero reducía drásticamente las metas y el presupuesto destinado a los programas alimentarios. Los movimientos en el tipo de cambio y las presiones inflacionarias del mercado interno, luego de la crisis financiera de diciembre de 1994, justificaban, desde la perspectiva oficialista, la decisión de liberar precios y retirar los subsidios de los productos básicos, así como reducir el gasto público en materia de programas sociales. En este escenario aparece, en agosto de 1997, el Programa de Educación, Salud y Alimentación (Progresa), ${ }^{3}$ como una variante adicional que sustituía al anterior y redirigía las metas y funcionamiento técnico en favor del combate a la pobreza extrema en las zonas rurales, así como el "fortalecimiento de la seguridad alimentaria" mediante el acceso a alimentos inocuos y de calidad nutricional a un mayor número de familias, solo que ahora bajo un esquema de subsidios directos. Algunas cifras indican que durante el bienio de 1998-1999 el Progresa gastó 3769,6 millones de pesos en ayuda alimentaria directa, es decir, el $31 \%$ del total del presupuesto de este programa gubernamental (Scott, 1999).

\footnotetext{
3 El Progresa representó una innovación de combate a la pobreza extrema rural en México, y posiblemente en el ámbito internacional, al combinar simultáneamente, para cada familia beneficiada, apoyos en tres áreas críticas y complementarias en la formación de capital humano básico, las cuales son la educación, la salud y la alimentación (Scott, 1999).
} 
De nueva cuenta, las recomendaciones de la FAO estipuladas en el informe sobre seguridad alimentaria de 1996 fueron importantes para el diseño de la política social del gobierno de Zedillo. No obstante, tuvieron escasa repercusión, por las debilidades de un modelo de política económica que veía en los subsidios indirectos un mecanismo de transferencia de ingreso que incidiría en la falta de incentivos a la producción, en el abasto y el encarecimiento de alimentos y, por lo tanto, generaría distorsiones en la economía de mercado, así como en el equilibrio fiscal (Meseguer, 1998). De acuerdo con ese razonamiento, siempre sería mejor otorgar directamente el subsidio en dinero a las familias pobres, antes que destinar subsidios generalizados a los productos, o dirigidos mediante programas de asistencia alimentaria, mismos que, según sus promotores, han probado ser ineficientes por sus costos operativos, su escasa coordinación técnica y la falta de acciones efectivas.

El seguimiento del nuevo esquema de subsidios persistió en la siguiente administración del presidente Vicente Fox. Su política social adoptó al Progresa como su hijo predilecto, y a partir de marzo del 2002 lo rebautizó con el nombre de Oportunidades. De acuerdo con el cuarto informe de gobierno 2004, el Programa Oportunidades se concibió como "uno de los principales instrumentos del Gobierno Federal para promover el desarrollo de las familias que viven en condiciones de pobreza de capacidades y para romper su transmisión intergeneracional, a través de acciones integrales y coordinadas en sus tres componentes: educación, salud y alimentación" (Poder Ejecutivo Federal de México, 2004, p. 32). Aparentemente, parte de sus propósitos centrales era respaldar el acceso efectivo a una alimentación adecuada y apoyar el gasto familiar de la población con los mayores índices de marginación, con énfasis en los grupos vulnerables.

De manera específica, las acciones contra la pobreza alimentaria se concretaron en la Estrategia Integral de Asistencia Social Alimentaria; Suplementos Alimenticios del Programa Oportunidades; Programa de Abasto Social de Leche; Programa de Abasto Rural, y Programa de Apoyo Alimentario. Al cobijo de estos programas, las acciones enfocadas en favorecer la seguridad alimentaria del país estuvieron igualmente concebidas en los términos sugeridos por el informe de la FAO de 1996. En este se insistía que la seguridad alimentaria solo sería posible cuando todas las personas tengan acceso físico y económico a suficientes alimentos inocuos y nutritivos 
para satisfacer sus necesidades y preferencias alimenticias, a fin de llevar una vida activa y sana. Así, la seguridad alimentaria se consigue cuando se garantiza la disponibilidad de alimentos, cuando el suministro es estable y cuando todas las personas los tienen a su alcance (FAO, 1996b).

Bajo estos lineamientos, la representación de México ante la FAO, junto con la Subsecretaría de Desarrollo Rural de la Secretaría de Agricultura, Ganadería, Desarrollo Rural, Pesca y Alimentación (Sagarpa), impulsó el Programa Especial para la Seguridad Alimentaria. ${ }^{4}$ Este fue concebido como una herramienta de apoyo técnico al Gobierno Federal para desarrollar metodologías y procedimientos orientados a impulsar la producción agrícola, mejorar el acceso de la población a los alimentos y crear condiciones aptas para incentivar la inversión pública y privada en el sector agroalimentario. Según la Sagarpa, su finalidad era la promoción de la productividad agropecuaria y el aumento de los ingresos de los pequeños productores del sector agropecuario para contribuir a mejorar la seguridad alimentaria a escala familiar y nacional, con atención especial a las poblaciones de más alta marginación del país, ubicadas en 250 microrregiones. ${ }^{5}$

Era evidente, sin embargo, que dicho programa pretendía reivindicar una política social de mayor alcance respecto a sus predecesores, y con ello obtener el reconocimiento de los organismos multinacionales de crédito y buscar consensos entre las fuerzas políticas del país de cara a las futuras elecciones. De hecho, a finales del sexenio del presidente Fox, la política social de gobierno, en especial el programa Oportunidades, fue calificado por el Banco Mundial y el Fondo Monetario Internacional como la "prueba creíble" de cómo un programa de tal naturaleza podía asegurar la continuidad del cambio sexenal. El informe del Banco Mundial titulado Development and the Next Generation 2007 atribuía el éxito del programa a la cantidad de recursos destinados al combate a la pobreza; pero, sobre todo, al impacto decisivo de los subsidios otorgados en dinero a las familias, así como a los

4 El Programa Especial para la Seguridad Alimentaria inició en 1994 con quince países de bajos ingresos y déficit de alimentos. Fue ratificado por los jefes de Estado y de Gobierno durante la Cumbre Mundial sobre la Alimentación que se celebró en Roma en noviembre de 1996.

5 De acuerdo con la Oficina Regional de la FAO para América Latina y el Caribe, el Programa Especial para la Seguridad Alimentaria inició como una estrategia piloto solo en siete estados de la república mexicana a partir del 2003: Michoacán, Aguascalientes, Jalisco, Yucatán, Guanajuato, Puebla y Estado de México, y cubría un total de doce municipios de alta y muy alta marginación, de los cuales cuatro son de mayoría indígena. 
"esfuerzos del Ejecutivo por romper el régimen burocrático" y dar seguridad de que los depósitos fueran entregados a la gente más necesitada.

Incluso en el medio académico, algunas opiniones destacan la difusión y reconocimiento internacional que fueron adquiriendo los programas Oportunidades y Progresa, al grado de ser considerados un ejemplo de política social que debía ser imitada por otros países, particularmente los de América Latina. Su aceptabilidad se atribuye, entre otras razones, tanto a la colaboración y participación estrecha de académicos e investigadores en el diseño, instrumentación, seguimiento y evaluación como en la decisión política de funcionarios de llevar a la práctica políticas conjuntas con la sociedad civil (Cortés, Escobar y González, 2005).

El programa Oportunidades, sin embargo, como sus antecesores Progresa y Solidaridad, no ha resistido la crítica de una parte de la clase política mexicana, que los considera un instrumento del aparato de Estado y del partido en el poder para coaccionar el voto y la participación ciudadana durante los periodos electorales. De hecho, la campaña electoral del 2006 fue una de las más ríspidas en señalamientos a la Secretaría de Desarrollo Social y a la Presidencia de la República por utilizar y dirigir los recursos de dicho programa con fines partidistas. Distintos analistas políticos (Cervantes, 2006; Delgado, 2006) señalaron la incorporación de miles de familias al programa Oportunidades en los últimos meses de gobierno del presidente Fox para favorecer al candidato del Partido Acción Nacional. Asimismo, señalaban el uso del padrón de afiliados de dicho programa para conseguir apoyos que requerían municipios y localidades urbanas y rurales, además de elaborar discursos de campaña con base en las necesidades de cada grupo demográfico. Incluso evaluaciones oficiales recientes, como la efectuada por la Auditoría Superior de la Federación al Programa de Apoyo Alimentario de Diconsa, incluido en el programa Oportunidades dependiente de la Secretaría de Desarrollo Social, encontró que este atendió apenas a un $18 \%$ de las comunidades de alta marginación en el país. Incorporó a familias que no necesitaban el apoyo y entregó recursos a hogares que no contaban con los requisitos de elegibilidad dispuestos en las reglas de operación del programa, lo que dejó fuera al 17\% de los beneficiarios objetivo, además 
de que concentró los recursos en solo cuatro estados de la república: Veracruz, Chiapas, Oaxaca y Guerrero. ${ }^{6}$

Al finalizar el sexenio de Fox, y con ello, la continuación del partido de la alternancia en el poder encabezado por Felipe Calderón, se prosiguió con estrategias similares para el combate a la pobreza. Así, se potenció de nueva cuenta el programa Oportunidades, que incrementó su cobertura en cerca de 6.500.000 familias. Además, se creó de emergencia el PAL, el cual tuvo una cobertura de 700.000 familias. No obstante, en esta administración, al igual que la de su predecesor, se enfrentó un escenario económico complejo caracterizado por la desaceleración económica estadounidense, que impactó de manera negativa en la economía nacional, al grado de registrar un decrecimiento negativo del producto interno bruto del 6,5\% para el 2009.

El manejo político del programa Oportunidades es, como se puede apreciar, solo otro ejemplo de la historia sexenal de nuestro país, que demuestra la fuerza que las instituciones del Estado y el Ejecutivo han venido ejerciendo en la organización, control y acceso de los recursos alimentarios y financieros destinados a la asistencia social. Detrás de ello están las relaciones de poder que en diversas coyunturas políticas han condicionado parte de la situación alimentaria de la sociedad mexicana y la han sometido a intereses económicos aparentemente distributivos, pero que en la práctica aparecen desligados de los propósitos de justicia social y soberanía alimentaria.

En el sexenio actual del gobierno de Enrique Peña Nieto (2012-2018), la apuesta para enfrentar la seguridad alimentaria inicia con objetivos aparentemente más ambiciosos. Esta se engloba en un proyecto de política social mucho más amplio llamado Cruzada Nacional contra el Hambre. En este se contempla:

[...] erradicar el hambre a partir de una alimentación y nutrición adecuada de las personas en pobreza multidimensional extrema y carencia de acceso a la alimentación; eliminar la desnutrición infantil aguda y mejorar los indicadores de peso y talla de la niñez; aumentar la producción 
de alimentos y el ingreso de los campesinos y pequeños productores agrícolas; minimizar las pérdidas post-cosecha y de alimentos durante su almacenamiento, transporte, distribución y comercialización, y promover la participación comunitaria para la erradicación del hambre (Cruzada Nacional contra el Hambre, 2013).

Durante sus dos primeros años de operación, el citado proyecto da continuidad al programa Oportunidades de las administraciones pasadas y, en septiembre de 2014, se sustituye por uno nuevo conocido como Prospera. A diferencia de los programas anteriores, con este se pretende instrumentar, entre otros aspectos, un marco específico regulatorio de política social aparentemente mucho más efectivo enfocado en las comunidades pobres y en mayor situación de pobreza que estén en posibilidades de generar ingresos propios mediante la reactivación de sus pequeñas parcelas, huertos de traspatio y actividades comerciales en pequeña escala. Sus resultados están por verse en lo que resta del sexenio.

\section{Marco constitucional de la política alimentaria en México}

Los términos de la Constitución Política de los Estados Unidos Mexicanos, sobre los derechos para el desarrollo social, la alimentación, la educación, la salud, la vivienda, el disfrute de un medio ambiente sano, el trabajo y la seguridad social se encuentran contenidos en diversos artículos que sustenta el respeto a la dignidad y el goce pleno de la vida de los mexicanos. En lo que a alimentación respecta, hasta el 2011, tanto el artículo $4 .^{\circ}$ como el $2{ }^{\circ}$ se refieren a dos grupos vulnerables de la sociedad que son los niños y los grupos indígenas. En el artículo $4{ }^{\circ}$ se establece el derecho de los niños y niñas para la satisfacción de sus necesidades de alimentación, entre otras necesidades, para su desarrollo integral. En el mismo sentido, en el artículo 2. ${ }^{\circ}$, fracción III del apartado B, se hace hincapié en la creación de instituciones y el diseño de políticas, que los tres órdenes de gobierno deben garantizar para promover la igualdad de oportunidades, principalmente para apoyar la nutrición de los indígenas mediante programas de alimentación, en especial para la población infantil, incluso en la fracción 
VIII se refiere especialmente la atención en la alimentación, a través de la política social, de las familias de los migrantes de origen indígena.

Sin embargo, como parte del reconocimiento del problema alimentario existente en el país, en el artículo $4 .^{\circ}$ y en el 27 se hicieron las siguientes adiciones: "toda persona tiene derecho a la alimentación nutritiva, suficiente y de calidad. El estado lo garantizará". En el artículo 27 se adiciona que "El desarrollo rural integral y sustentable a que se refiere el párrafo anterior también tendrá entre sus fines que el Estado garantice el abasto suficiente y oportuno de los alimentos básicos que la ley establezca".

Después de estos dos artículos, solo el último párrafo del artículo 16 refiere al derecho de los militares a exigir alimentos, además de alojamiento, bagajes y otras prestaciones, en tiempo de guerra. Es importante mencionar que no hay otro artículo en el cual se establezca de manera expresa el derecho de todos los mexicanos al acceso a los alimentos.

\subsection{El Plan Nacional de Desarrollo 2007-2012}

Debido a que la Constitución sirve como marco general de los derechos y obligaciones del Estado hacia sus ciudadanos, desde la presidencia del general Lázaro Cárdenas del Río se instauraron los PND, los cuales contienen la visión, los ejes de política y las acciones que va a desarrollar el gobierno en turno. Es decir, al ser la Constitución un documento general, para atender los problemas del país se requiere el diseño e implementación de políticas públicas que se encuentren definidos en un sistema nacional de planeación. Este sistema de planeación tiene sustento constitucional en el artículo 25, donde se establece la rectoría del Estado sobre el desarrollo nacional para que los individuos puedan ejercer plenamente los derechos contenidos en la Constitución. De la misma forma, el artículo 26 define que esta planeación debe ser democrática de manera que se involucren los diversos sectores sociales para incorporar sus aspiraciones y demandas. Corresponde tanto al poder Ejecutivo como al poder Legislativo su elaboración y se faculta al primero para que establezca los procedimientos de participación y consulta con esos sectores sociales, así como los criterios para la formulación, la instrumentación, el control y la evaluación del plan y los programas de desarrollo. En el mismo artículo se establece que el Estado contará con un Sistema Nacional de Información Estadística y Geográfica, 
para obtener datos oficiales tanto de la Federación como de los estados, municipios y el Distrito Federal. Estos datos son la base del sistema de planeación de los tres órdenes de gobierno (Ejecutivo, Legislativo y Judicial), pues a partir de ellos se identifica la población objetivo de los programas que implementan cada uno de ellos.

Del sistema de planeación democrática se desprenderán no solo los programas de la Administración Pública Federal, sino que las propias planeaciones de los estados y municipios tienen que apegarse a sus principios y estar coordinados por el sistema nacional. Una de las herramienta centrales del Sistema Nacional de Planeación es el PND, que entre sus objetivos sobre el tema que nos ocupa en este trabajo se encuentran reducir la pobreza extrema y asegurar la igualdad de oportunidades para que todos los mexicanos tengan garantizada la alimentación, entre otros derechos, para que tengan un mejor nivel de calidad de vida, tal y como se establece en la Constitución.

Entre sus estrategias de política pública se encuentran el logro del desarrollo humano de las personas a partir de la familia y para ello el plan contempla la implementación de políticas que contribuyan a fortalecer a la familia en relación con el acceso a los alimentos. En el PND se reconoce la importancia de las actividades agrícolas como fundamentales para la producción de los alimentos que se consumen en el país; por eso al sector rural, que incluye las actividades agropecuarias y pesqueras principalmente, se les presta especial atención, pues una cuarta parte de los mexicanos vive en el campo y enfrenta graves condiciones de pobreza.

Otro de los aspectos que el PND reconoce es la baja explotación de los recursos pesqueros a pesar de las grandes extensiones de costas que poseen un potencial pesquero diverso y de grandes volúmenes de captura; sin embargo, han faltado mecanismos adecuados para su aprovechamiento, sobre todo para que ello repercuta a favor de la alimentación y la mejora de las condiciones de quienes se dedican a estas actividades.

Asimismo, se definen una serie de objetivos y estrategias que tienen como finalidad elevar el desarrollo humano y patrimonial de los mexicanos que viven en las zonas rurales y costeras, a través de programas como el Programa Especial Concurrente, el cual es coordinado por la Sagarpa, en colaboración con otras dependencias que inciden en el campo. Al mismo tiempo se pretenden otros objetivos como la diversificación de productos, 
el fomento del consumo de productos nacionales en el mercado interno, la participación activa de mujeres y jóvenes en las actividades agrícolas y pesqueras, la modernización del sector agrícola y pesquero y, principalmente, la promoción de la seguridad alimentaria, mediante el fomento de cultivos y productos básicos y el fortalecimiento de su comercialización mediante la promoción y el ordenamiento de mercados.

En el marco de los acuerdos internacionales como el Tratado de Libre Comercio con América del Norte, el PND prevé un seguimiento puntual a los programas dirigidos a elevar la competitividad del maíz, del fríjol, del azúcar y de la leche, como productos básicos de la dieta mexicana. En este sentido, se tiene prevista la continuidad del programa Procampo, por lo menos hasta que termine el presente sexenio. En materia de coordinación, la estrategia 11.3 refiere la cooperación con los gobiernos estatales para implementar políticas enfocadas al medio rural.

Uno de los problemas más graves en el campo mexicano es la pobreza. Para esto, el eje 3 se propone la igualdad de oportunidades a través de la superación de la pobreza, donde se identifican los tres tipos de pobreza por ingreso: alimentaria, de capacidades y patrimonial. Las estrategias consisten en fortalecer los programas existentes con mayores recursos, para que las familias que los necesiten puedan mejorar la alimentación, la salud y la educación de sus hijos, esto con el sustento de las evaluaciones nacionales e internacionales sobre dichos programas.

Parte de estas estrategias consiste en impulsar un mayor desarrollo de los municipios más pobres, mediante la focalización de recursos y la coordinación de esfuerzos entre el Gobierno Federal y los demás órdenes de gobierno. El PND destaca que si no se cuenta con comida, techo, electricidad, salud, educación, etc., la población en desventaja difícilmente podrá ampliar sus capacidades para remontar la pobreza; por eso se busca fortalecer los programas de apoyo alimentario vigentes, tratando de mejorar y unificar, bajo criterios comunes, las vertientes de alimentación y nutrición, así como otras líneas de acción de las instituciones públicas que atienden estos problemas. Además, se busca coordinar efectivamente las instituciones federales para propiciar acuerdos con los estados, municipios y los sectores social y privado, a fin de enfrentar juntos el problema y proveer los apoyos a la población más necesitada. Destaca la meta de abatir la desnutrición infantil y materna del país. 
En resumen, en este apartado se pudo conocer brevemente los objetivos y estrategias del PND en dos grandes aspectos que son esencia del presente trabajo: el sector rural y la superación de la pobreza alimentaria. Destaca la preocupación y reconocimiento que hace el PND a la producción de algunos alimentos altamente consumidos por el mexicano, así como algunas estrategias orientadas a fortalecerla y al sector rural. Sin embargo, el PND parece concebirse más como un instrumento de "comunicación social" que como un plan detallado de acciones, mecanismos, herramientas y presupuestos que sirvan de guía para la consecución de los objetivos planteados por este. Es decir, el PND se asemeja más a un ejercicio de planeación estratégica que incluye misión y visión de país, así como algunas acciones y estrategias; pero que en la parte meramente instrumental carece de lo necesario para una implementación efectiva, al menos como viene planteado en el documento.

\section{Competencias de los órdenes de gobierno sobre la política alimentaria en las leyes General de Desarrollo Social y de Desarrollo Rural Sustentable}

Una vez vistos los temas relacionados con este tema en el PND, analizaremos dos de las principales leyes que regulan, por una parte, la política social dentro de la cual se encuentra la atención a la alimentación y, por otra, la encargada directamente de la seguridad y soberanía alimentaria. Estas leyes son la Ley General de Desarrollo Social (LGDS) y la Ley de Desarrollo Rural Sustentable (LDRS). La primera tiene mayor relación con la Secretaría de Desarrollo Social, la cual -a pesar de no dedicarse exclusivamente al tema alimentario- opera diversos programas para tal fin, como el PAL, el Programa para el Abasto de Leche, el Programa de Abasto Rural y Oportunidades (que apoya la educación, salud, alimentación, pago de la energía eléctrica y adultos mayores). La LDRS, a su vez, se relaciona principalmente con la Sagarpa y se entiende que esta se hará cargo de la coordinación de la ejecución de dicha ley junto con otras secretarías que tienen programas o fines vinculados con la alimentación en el país. 


\subsection{La alimentación como parte de la política social}

Buena parte de las generalidades planteadas por la constitución y el PND en lo referente al sistema de planeación se detalla en mayor medida en la LGDS. En consecuencia, en su primer artículo, fracción III, señala que la prioridad es establecer un Sistema Nacional de Desarrollo Social en el que participen los gobiernos municipales, las entidades federativas (equivalentes a las provincias sudamericanas) y la federación. En adición, en el mismo artículo $1 .^{\circ}$, fracción IV, se determinan las competencias de los gobiernos municipales, de las entidades federativas y del Gobierno Federal en materia de desarrollo social, así como las bases para la concertación de acciones con los sectores social y privado.

La orientación de la LGDS es establecer concurrencias, responsabilidades y, sobre todo, facilitar la coordinación entre los tres ámbitos de gobierno en aras de contribuir a un efectivo desarrollo social. Para el desarrollo social es sumamente trascendental dejar en claro cuáles son los aspectos comprendidos como fundamentales para lograrlo. En correspondencia, el artículo 6 señala: "Son derechos para el desarrollo social, la educación, la salud, la alimentación, la vivienda, el disfrute de un medio ambiente sano, el trabajo y la seguridad social y los relativos a la no discriminación en los términos de la Constitución Política de los Estados Unidos Mexicanos" (figura 1).

En concreto, para la LGDS los derechos fundamentales de todo ser humano como: la educación, la vivienda, la salud y la alimentación, etc., se configuran como los componentes principales que busca generar el Sistema Nacional de Desarrollo Social. Adicionalmente, el artículo 9 abunda sobre las responsabilidades de los gobiernos al apuntar que:

Los municipios, los gobiernos de las entidades federativas y el Poder Ejecutivo Federal, en sus respectivos ámbitos, formularán y aplicarán políticas compensatorias y asistenciales, así como oportunidades de desarrollo productivo e ingreso en beneficio de las personas, familias y grupos sociales en situación de vulnerabilidad, destinando los recursos presupuestales necesarios y estableciendo metas cuantificables.

De antemano, se observa que en la LGDS se plantean dos tipos de responsabilidades y facultades para los tres niveles de gobierno existentes en el país: 


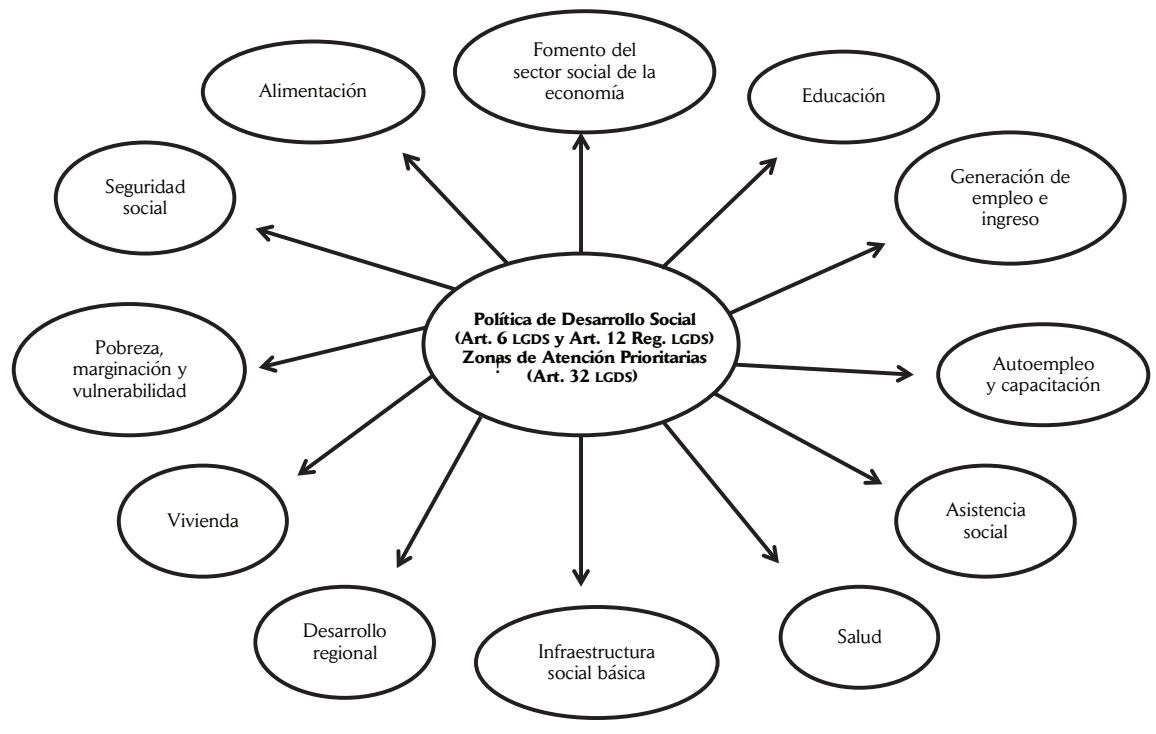

Fuente: Elaborado por el autor con base en la Ley General de Desarrollo Social (2009).

1. Responsabilidades y facultades exclusivas: son aquellas que le corresponden exclusivamente al gobierno dentro de su espacio territorial. Para este tipo de responsabilidades y facultades se aplican normas y leyes que deben ser cumplidas en los espacios territoriales correspondidas a los tres ámbitos de gobierno.

2. Responsabilidades y facultades compartidas: son aquellas que debido a su importancia y, sobre todo, a que se configuran como trascendentales para el desarrollo del país se plantean de manera compartida entre los tres ámbitos de gobierno. Por ejemplo, asegurarse que toda la población posea la capacidad de acceder a alimento suficiente en todo momento es un objetivo de gran complejidad que exige la coordinación gubernamental a gran escala (figura 2).

Respecto a la aplicación, el artículo $4 .^{\circ}$ señala que será el Ejecutivo Federal el que aplique la LGDS por conducto de sus dependencias y organismos; así mismo, le corresponde la aplicación a los poderes ejecutivos de las entidades federativas (estados de la República Mexicana) y a los municipios en el ámbito de sus respectivas competencias, y las que les competen, 


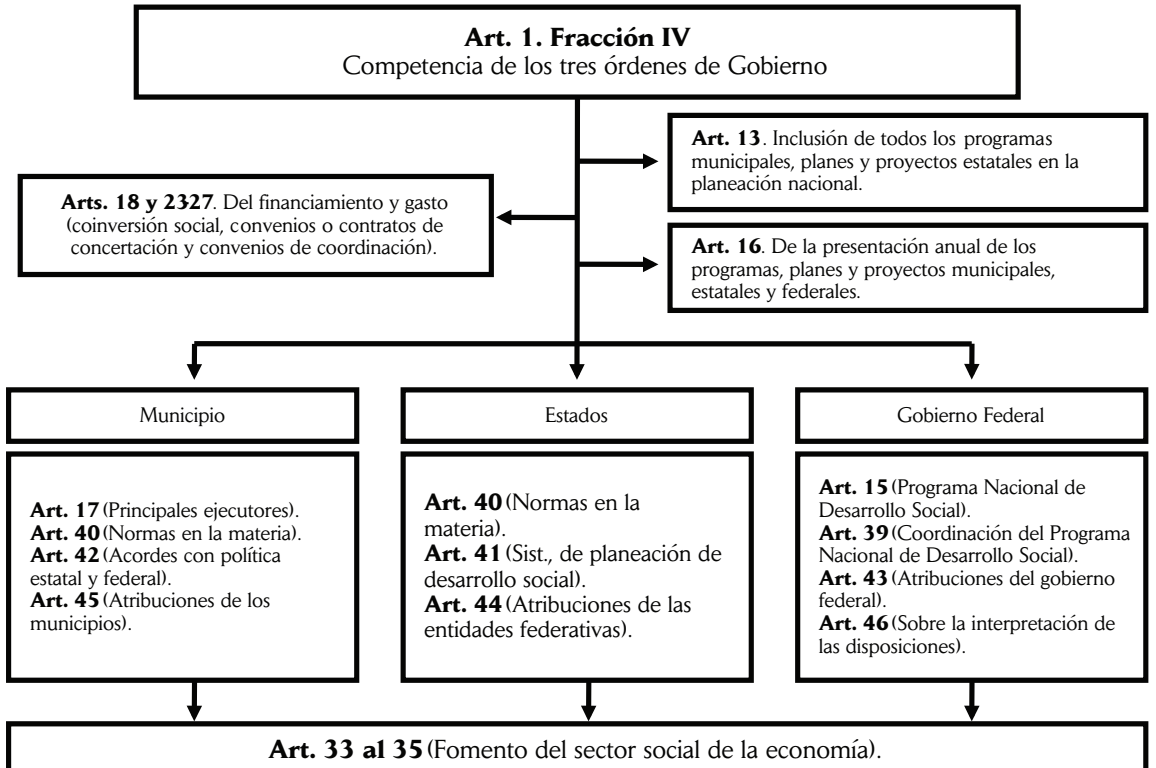

Fuente: Elaborado por el autor con base en la Ley General de Desarrollo Social (2009).

de acuerdo con sus atribuciones, al Poder Ejecutivo. En este sentido, la fracción IV del artículo 1. ${ }^{\circ}$ señala que esta ley determina qué corresponde a los gobiernos municipales, a las entidades federativas y al Gobierno Federal en materia de desarrollo social; también determina las bases para la concertación de acciones con los sectores social y privado. Para ejecutar la política social es necesario el establecimiento de un Sistema Nacional de Desarrollo Social (fracción III, artículo 1. ${ }^{\circ}$ de las disposiciones generales), en el que participen los gobiernos municipales, de las entidades federativas y el federal. Este Sistema Nacional de Desarrollo Social, de acuerdo con el artículo 38 de la LGDS, es un mecanismo permanente de concurrencia, colaboración, coordinación y concertación de los tres órdenes de gobierno, así como de los sectores social y privado, en la formulación, ejecución e instrumentación de programas, acciones e inversiones en la materia que promueven que todo esté acorde a la Política Nacional de Desarrollo Social.

En el marco del federalismo y los procesos de nuevas formas de gobernar, impulsa la desconcentración y descentralización de los recursos y acciones para el desarrollo social, la transparencia y rendición de cuentas. 
La coordinación del Sistema Nacional estará a cargo de la Secretaría de Desarrollo Social. Lo conformarán los siguientes órganos: la Comisión Nacional, la Comisión Intersecretarial (conformada por representantes de diversas secretarías federales), el Consejo Consultivo (conformado por ciudadanos de reconocido prestigio en los sectores privado y social) y el Consejo Nacional de Evaluación de la Política de Desarrollo Social (integrado por seis investigadores académicos y tienen la finalidad de evaluar los diversos programas de la política social). El artículo 47 establece que la Comisión Nacional se encargará de coordinar que los objetivos, las estrategias y las prioridades de la Política Nacional de Desarrollo Social se pueden llevar a cabo desde el ámbito de competencia de los órdenes de gobierno, o bien en concurrencia entre ellos o incluso en concertación con los sectores social y privado.

Como se menciona en el PND, los tres órdenes de gobierno tienen la facultad de formular y aplicar políticas compensatorias y asistenciales en beneficio de las personas y grupos vulnerables (artículo 9); sin embargo, todos deben estar alineados al PND (artículo 13). Sobre el financiamiento de la política de desarrollo social, el artículo 18 indica que por ningún motivo disminuirá el presupuesto destinado a estas actividades y la Cámara de Diputados deberá considerar, por lo menos, el presupuesto del año anterior para fijar el nuevo presupuesto. Además, de acuerdo con el artículo 25 de la Constitución, se podrán buscar otras fuentes de financiamiento por parte de los tres órdenes de gobierno utilizando los mecanismos de colaboración que se definen en el artículo 3 del reglamento de la LGDS para hacer partícipes a la sociedad civil y los sectores social y privado en las actividades de desarrollo social. Estos mecanismos son los siguientes: la coinversión social, que se refiere a la participación, conjunta o individual, de organizaciones de los sectores social y privado, mediante la aportación de recursos humanos, materiales o financieros en las acciones y programas de desarrollo social; los convenios o contratos de concertación, que aluden al otorgamiento de derechos a organizaciones o agencias del sector privado para la operación de ciertas actividades (programas o proyectos) de desarrollo social, y los convenios de coordinación, que se pueden dar entre los diferentes órdenes de gobierno para implementar políticas conjuntas de desarrollo social. En todos los casos, la Secretaría de la Función Pública será la institución encargada de vigilar la elaboración de los convenios y su cumplimiento. 
Aun cuando se considera al municipio como el principal ejecutor de la política social (artículo 17), el artículo 42 lo limita a alinear sus propias políticas a las políticas estatal y federal. En el mismo tenor, el artículo 45 establece las atribuciones y la emisión de normas (artículo 40) de los municipios sobre su política social, lo cual en la actualidad es muy difícil de llevar a cabo, pues muchos municipios siquiera tienen un plan de desarrollo municipal (Cabrero, 2005).

Respecto a los Estados, no hay mayor diferencia, pues llegan a servir como el orden de gobierno intermediario entre el Federal y Municipal en muchas de las atribuciones. Tienen la facultad de emitir normas en materia de desarrollo social (artículo 40) o crear su propio sistema de planeación (artículo 41), y sus atribuciones específicas se detallan en el artículo 44, pero finalmente también tienen que supeditarse a la planeación nacional.

El Gobierno Federal tiene la mayor responsabilidad tanto para el diseño como para la implementación de la política desarrollo social, pues coordina el Programa Nacional de Desarrollo Social (artículos 15 y 39), que no solo comprende las acciones de ese orden de gobierno, sino que también incluye, como ya se dijo, las acciones de los gobiernos estatales y municipales. El artículo 43 menciona las atribuciones del gobierno federal en la materia y, en caso de duda sobre las atribuciones con los otros órdenes, el gobierno Federal detenta la facultad de tomar la decisión (artículo 46) (figura 3).

De esta manera, en la LGDS se establecen los lineamientos generales en términos de coordinación, ejercicio de atribuciones y mecanismos de colaboración de la política social tanto para los órdenes de gobierno como para la participación de la sociedad civil, en la cual se considera la alimentación una de sus áreas de atención.

\subsection{Ley de Desarrollo Rural Sustentable}

Una de las leyes fundamentales para la política alimentaria es la LDRS, pues en ella se define la forma en que se incentiva y regulan las actividades agrícolas destinadas a la producción de alimentos y se fomenta el desarrollo del campo mexicano.

Por ello, en el artículo $3 .^{\circ}$, en la fracción XXVII, se utiliza el concepto de seguridad alimentaria como el abasto oportuno, suficiente e incluyente de alimentos a la población; mientras que el concepto de soberanía alimentaria 


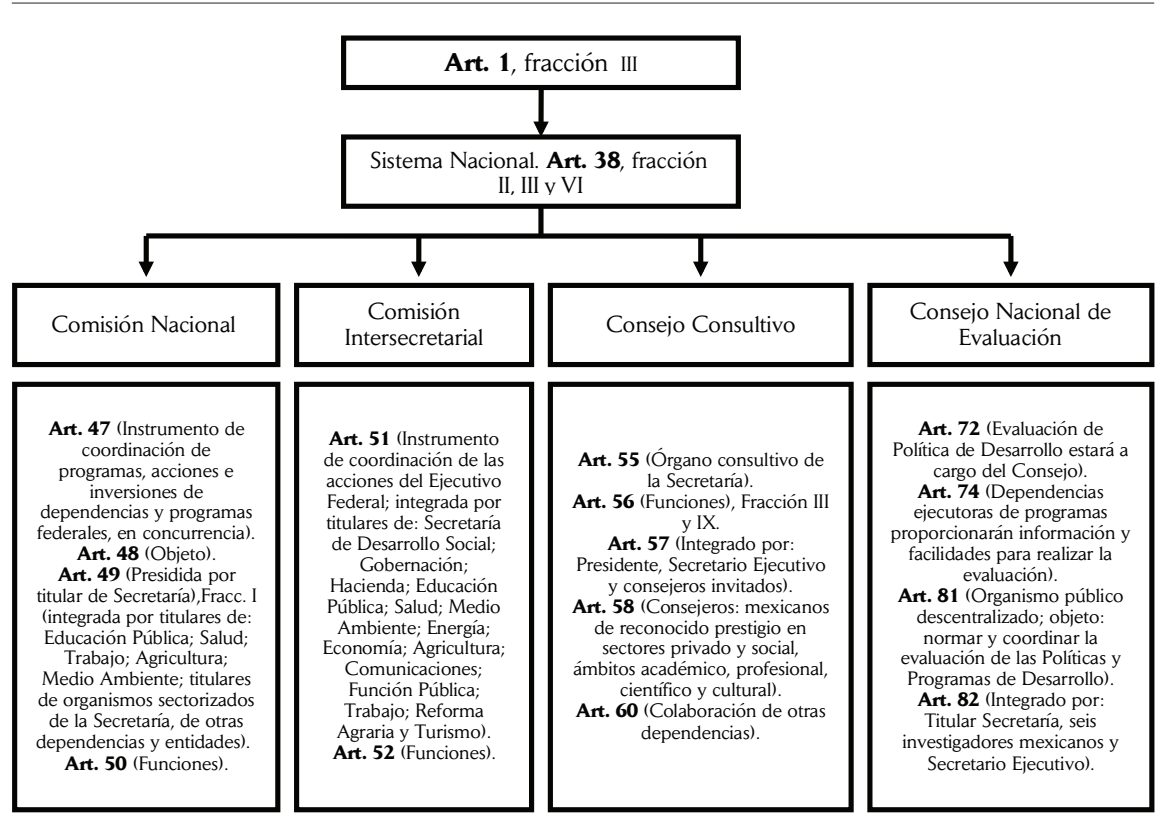

Fuente: Elaborado por el autor con base en la Ley General de Desarrollo Social (2009).

se encuentra en la fracción XXXII y se le entiende como la libre determinación del país en materia de producción, abasto y acceso de alimentos a toda la población, basada fundamentalmente en la producción nacional. Sin entrar a más detalles de cada uno de los conceptos, se hace una clara distinción entre ellos y en el artículo 5 . $^{\circ}$ se retoman ambos conceptos como uno de los objetivos del Estado, a través del Gobierno Federal, al diseñar e implementar políticas, acciones y programas para impulsar las actividades agropecuarias en el medio rural, en coordinación con los gobiernos estatales y municipales.

Al igual que en la LGDS, los convenios de colaboración entre los órdenes de gobierno para poner en práctica programas de apoyo para el desarrollo rural sustentable se ajustarán a los criterios del Plan Nacional de Desarrollo (artículo 23 de la LDRS), en el marco de respeto al federalismo y la descentralización en el establecidos. Mediante los convenios entre la federación y los estados se crearán los consejos para el Desarrollo Rural Sustentable, homologados al Consejo Mexicano, en los municipios, en los distritos de 
Desarrollo Rural y en las entidades federativas (artículo 24), los cuales serán las instancias de productores y demás agentes de la sociedad para participar en la planeación y distribución de los recursos destinados para tales actividades. La conformación de dichos consejos está determinada en los artículos 25 y 26, donde se menciona la participación de las autoridades estatales y municipales.

Las responsabilidades de cada uno de los órdenes de gobierno se definirán en los convenios, de acuerdo con el artículo 27 y lo referente a la administración y distribución de los recursos de la coordinación se establece en el artículo 28. En este último tema, la fracción V del artículo 191 refiere los apoyos que se otorgarán a los productores bajo el criterio de concurrencia entre los tres órdenes de gobierno y los propios beneficiarios, a fin de asegurar la corresponsabilidad entre el Estado y la sociedad para el uso eficiente de los recursos destinados a las actividades agropecuarias.

Acerca de la seguridad y soberanía alimentaria, el capítulo XVII de la LDRS establece que el Estado determinará las medidas para procurar el abasto de alimentos y productos básicos estratégicos a la población, al tiempo que promoverá su acceso a los grupos sociales menos favorecidos y dará prioridad a la producción nacional (artículo 178). A efectos de conocer cuáles son los productos básicos y estratégicos, el artículo 179 enumera los siguientes: maíz, caña de azúcar, fríjol, trigo, arroz, sorgo, café, huevo, leche, carne de bovinos, porcinos, aves y pescado; por lo tanto, el Gobierno Federal tiene la obligación de conducir su política agropecuaria, a fin de que los programas y acciones para el fomento productivo y el desarrollo rural sustentable, así como los acuerdos y tratados internacionales, propicien la inocuidad, la seguridad y la soberanía alimentaria, mediante la producción y abasto de los productos mencionados (artículo 180). Hay que destacar que hace hincapié en que corresponde al Gobierno Federal esta atribución y que no se considera la participación de los otros órdenes de gobierno en esta.

Para conocer los resultados de lo mencionado en el artículo anterior, se plantea la evaluación (artículo 181), por parte de los actores que intervienen en el desarrollo rural, lo cual incluye a todos los productores y agentes intervinientes en la integración de las cadenas productivas de alimentos (artículo 182). Una vez más, en el artículo 183 se establece que corresponde al Gobierno Federal acciones para los requerimientos de la seguridad y soberanía alimentaria, que implican aspectos de la producción, la prevención 
de riesgos, la atención a la demanda, la productividad y calidad, la comercialización nacional e internacional, las innovaciones tecnológicas, la capacitación, la elaboración de guías sobre prácticas en las diferentes etapas de las cadenas agroalimentarias, los programas y acciones de protección al medio ambiente y la aplicación de medidas de certidumbre económica, financiera y comercial que garanticen el cumplimiento de los programas productivos mencionados en el artículo 180.

\section{Conclusiones}

El problema de la alimentación adquiere cada vez más importancia no solo en el ámbito nacional, sino que es un problema que afecta a la mayoría de los países del mundo. El establecimiento de reglas claras es indispensable para que se puedan diseñar e implementar políticas públicas orientadas a la solución de dicho problema. En el caso de la legislación mexicana, es necesario realizar diversos ajustes que definan claramente una política alimentaria, ya que el Estado mexicano no tiene definida una política alimentaria con dependencias y programas que atiendan exclusivamente este problema, sino que encontramos programas aislados unos de otros en diversas dependencias que, al mismo tiempo, tienen que atender otros problemas de interés público como la vivienda, la educación, el fomento de actividades agropecuarias y pesqueras, el desarrollo rural, etc., como en el caso de la Secretaría de Desarrollo Social, lo cual impide una focalización en los asuntos prioritarios como la seguridad y soberanía alimentaria del país.

Como consecuencia de la indefinición de la política alimentaria, tampoco se tiene un objetivo claro, ya que dependiendo del gobierno de turno, los objetivos cambian de lograr la autosuficiencia alimentaria a la seguridad alimentaria o a la soberanía alimentaria que tienen diferencias que obligan a plantear la política considerando diferentes elementos. A todo esto se suma que el eje rector del desarrollo nacional, que es el PND, no contempla un apartado específico sobre los objetivos y estrategias de la alimentación en el país, sino que estas se definen en diferentes ejes, de acuerdo con los intereses y necesidades de los sectores dedicados a esta actividad (principalmente en la producción, distribución y comercialización de alimentos) o a la población objetivo (asegurar la alimentación de niños, mujeres e indígenas). 
Las limitadas atribuciones de los estados y municipios quedan demostradas en la operación de algunos de los programas y proyectos como los analizados en la última parte de este trabajo. En ellos ha quedado claro que el Gobierno Federal tiene la responsabilidad de casi todos los procesos que implican la ejecución de dichos programas; incluso se puede observar que las entidades federativas solo pueden ser medios para la ejecución de los recursos de las secretarías federales, como ocurre con el caso de la Sagarpa, a través de Procampo. En Oportunidades, las entidades federativas solo participan mediante los servicios educativos y de salud que, debido a la descentralización de estos servicios, tienen la obligación de proporcionar para cumplir con los objetivos del programa, pero no hay ninguna referencia sobre su participación en los asuntos alimentarios. Resulta más grave cuando se analiza el papel de los municipios, pues incluso la colaboración de estos se limita a las actividades de enlace y logística, como es el caso de Oportunidades, donde tienen que proporcionar el mobiliario para que los operadores del programa puedan realizar sus actividades.

Por ello solo a través de una mayor claridad en el marco regulatorio se podrán diseñar políticas públicas más efectivas que contribuyan de manera más decisoria a combatir el problema alimentario existente en México. No obstante, mientras no se tomen estas acciones, se continuarán realizando esfuerzos aislados que llevan al desperdicio de recursos, debido a la deficiente focalización de programas, estrategias mal concebidas y duplicidad de programas, por lo que la magnitud del problema demanda la exploración de soluciones alternativas que, al menos, generen mejores resultados que los obtenidos con el modelo actual, por lo que en función de lo anterior, bien vale la pena intentar.

\section{Referencias}

Barquera, S., Rivera-Dommarco, J. y Gasca-García, A. (2001). Políticas y programas de alimentación y nutrición en México. Salud Pública de México, 43(5), 464-477.

Cabrero Mendoza, E. (2005). Acción pública local. México: Fondo de Cultura Económica.

Centro de Estudios sobre Marginación y pobreza del Estado de México (2005). Pobreza infantil en México. Recuperado de http://www.edomexico.gob.mx 
Cervantes, J. (2006). El PAN compra el voto rural. Proceso (1541), 28-31.

Constitución Política de los Estados Unidos Mexicanos. Diario Oficial de la Federación. México, D. F. 05/02/1917.

Cortés, F., Escobar, A. y González, M. (2005). Método científico y política social. Guadalajara: Ciesas (mimeo).

Cruzada Nacional Contra el Hambre. (2013). Decreto por el que se establece el Sistema Nacional para la Cruzada Contra el Hambre. Diario Oficial de la Federación. México.

Delgadillo, J. y Cortez, H. (2003). Seguridad alimentaria y bienestar social. En F. Torres (Coord.), Seguridad alimentaria: seguridad nacional (pp. 173-202). México: Plaza y Valdés.

Delgado, A. (2006). Calderón-Presidencia: complicidad electoral. Proceso (1535), 22-24.

Food and Agriculture Organization (2006). Informe de políticas. Recuperado de http://www.sagarpa.gob.mx/v1/pesa/index.html

Huerta, A. (1987). La economía mexicana más allá del milagro. México: Cultura Popular.

Ley de Asistencia Social. Diario Oficial de la Federación. México, D. F. 02/09/2004. Ley de Desarrollo Rural Sustentable. Diario Oficial de la Federación. México, D. F. 07/12/2001.

Ley de Productos Orgánicos. Diario Oficial de la Federación. México, D.F. 07/02/2006. Ley de Promoción y Desarrollo de los Bioenergéticos. Diario Oficial de la Federación. México, D. F. 01/02/2008.

Ley Federal de Producción, Certificación y Comercio de Semillas. Diario Oficial de la Federación. México, D. F. 15/06/2007.

Ley Federal de Sanidad Animal. Diario Oficial de la Federación. México, D. F. 25/07/2007.

Ley Federal del Impuesto al Valor Agregado. Diario Oficial de la Federación. México, D. F. 11/12/2013.

Ley Federal del Impuesto sobre la Renta. Diario Oficial de la Federación. México, D. F. 11/12/2013.

Ley General de Desarrollo Forestal Sustentable. Diario Oficial de la Federación. México, D. F. 25/02/2003.

Ley General de Desarrollo Social. Diario Oficial de la Federación. México, D. F. 20/01/2004.

Ley General de Pesca y Acuacultura Sustentables. Diario Oficial de la Federación. México, D. F. 24/07/2007. 
Ley General de Salud, México. Diario Oficial de la Federación. México, D. F. 07/02/1984.

Meseguer, X. (1998). La política social alimentaria y nutricional en México. El Debate Nacional, México, 207-228.

National Family Farm Coalition (NFFC), (2008). National Family Farm Coalition. Recuperado de http://www.nffc.net

Organización de las Naciones Unidas para la Alimentación y la Agricultura (FAO), (1996a). Contexto sociopolítico y económico general para la seguridad alimentaria en los niveles nacional, regional y mundial. Roma: FAO-ONU.

Organización de las Naciones Unidas para la Alimentación y la Agricultura (FAO), (1996b). Declaración de Roma sobre la Seguridad Alimentaria Mundial y el Plan de Acción de la cumbre mundial. Documento procedente de la Cumbre Mundial sobre la Alimentación, Roma, Italia.

Ortiz Gómez, A., Vázquez García, V. y Montes Estrada, M. (2005). La alimentación en México: enfoques y visión a futuro. Estudios Sociales. Revista de Investigación Cientifica, XIII(25), 7-34.

Plan Nacional de Desarrollo 2007-2012. Felipe Calderón Hinojosa. Diario Oficial de la Federación. México, D. F. 01/01/2007.

Poder Ejecutivo Federal de México (2004). Cuarto informe de ejecución 2004 del Plan Nacional de Desarrollo 2001-2006. Recuperado de http://pnd.presidencia. gob.mx/pdf/2004/M031-048.pdf

Presidencia de la República de México (1980). Sistema alimentario mexicano. México: Oficina de Asesores de la Presidencia.

Reglas de Operación de los programas de la Secretaría de Agricultura, Ganadería, Desarrollo Rural, Pesca y Alimentación (s. f.). Recuperado de http://www. sagarpa.gob.mx/programas/Documents/RO_2011.pdf

Reglas de Operación del Programa de Abasto Rural (PAR), (s. f.). Recuperado de http://www.diconsa.gob.mx/images/swfs/paayar/reglas_par_2011.pdf

Reglas de Operación del Programa de Apoyo Alimentario (PAL), (s. f.). Recuperado de http://www.oportunidades.gob.mx/Portal/wb/Web/reglas_de_operacion_pal_2011

Reglas de Operación del Programa de Apoyos para el Campo (Procampo), (s. f.). Recuperado de http://www.sagarpa.gob.mx/quienesomos/InformacionDelSector/MarcoJuridico/Lists/Marco\%20Jurdico/Attachments/6/reglas.pdf Reglas de Operación del Programa Desarrollo Humano Oportunidades (s. f.). Recuperado de http://www.oportunidades.gob.mx/Portal/wb/Web/reglas_ de_operacion_oportunidades_2011 
Scott, J. (1999). Análisis del Programa de Educación, Salud y Alimentación (Progresa). México: CIDE.

Suárez, B. y Pérez-Gil, F. (1999).La modernización del campo y la alimentación: un recuento de los últimos años, 1982-1996. En L. M. Espinoza (Coord.), Sector agropecuario y alternativas comunitarias de seguridad alimentaria y nutrición en México. México: UAM/CECIPROC/INNSz/Plaza y Valdés.

Vázquez, V., Ortiz, A. y Montes, M. (2005). La alimentación en México: enfoques y visión a futuro. Estudios Sociales, 13(25), 7-34.

Zapata, F. (2005).Tiempos neoliberales en México. México: El Colegio de México. 
